\title{
Plastic flows and strain-induced alpha to omega phase transformation in zirconium during compression in a diamond anvil cell: Finite element simulations
}

\author{
Biao Feng ${ }^{1} \quad$ and Valery I. Levitas ${ }^{2, *}$
}

1) Department of Aerospace Engineering, Iowa State University, Ames, Iowa 50011, USA

2) Departments of Aerospace Engineering, Mechanical Engineering, and Material Science and Engineering, Iowa State University, Ames, Iowa 50011, USA

\begin{abstract}
Coupled plastic flows and the strain-induced $\alpha \rightarrow \omega$ phase transformation (PT) in a zirconium sample under compression in a diamond anvil cell are investigated using finite element method (FEM). The PT is treated as strain-induced rather than pressure-induced and the previously developed model for strain-induced PTs is utilized. Very heterogeneous fields of stress tensor, accumulated plastic strain, and concentration of the $\omega$ phase are obtained for different applied loads. The PT starts at the center of a sample when pressure exceeds the minimum pressure $p_{\varepsilon}^{d}=1.7 \mathrm{GPa}$, below which a direct strain-induced PT to a high pressure phase cannot occur, and it propagates from the center to the periphery with an increasing load. Even at the maximum pressure of $7 \mathrm{GPa}$, the PT is not completed everywhere. With an increasing load, the pressure and pressure gradient along the radial direction significantly increase in the two-phase region due to the much larger yield strength of the $\omega$ phase. This in turn promotes transformation and produces a positive mechanochemical feedback. Obtained results are utilized for the interpretation of published experimental data on pressure-, stress-, and strain-induced $\alpha \rightarrow \omega$ PTs in $\mathrm{Zr}$ and Titanium (Ti) and $\alpha \rightarrow \beta$ and $\omega \rightarrow \beta$ PTs in $\mathrm{Zr}$ under compression and high pressure torsion. This includes correcting the reported minimum pressures for these transformations by a factor of 3 to 6 due to the stress heterogeneity, the effect of transmitting media, the pressure hysteresis, and the reversibility of the transformation.
\end{abstract}

Keywords: Strain-induced phase transformations, Zirconium, High pressure, Diamond anvil cell, Plasticity.

\footnotetext{
* Corresponding author.

Email address for Valery Levitas: vlevitas@iastate.edu

Email address for Biao Feng: fengbiao11@gmail.com
} 


\section{Introduction}

The diamond anvil cell (DAC) is widely used in the field of high pressure research and it allows an in-situ study of phase transformations (PTs) under high pressure by using advanced diagnostics such as optical, Raman, and $x$-ray techniques [1-4]. Within a liquid medium, the sample is subjected to a hydrostatic load and PTs in the sample are pressure-induced. Without hydrostatic medium, or above the solidification pressure of transmitting medium, a sample in a DAC is subjected to nonhydrostatic stresses or stress tensor. When a large, irreversible reduction of the thickness takes place, material is subjected to large plastic deformation. Experiments in rotational Bridgman anvils $[5,6]$ and rotational DAC show that plastic deformation leads to a significant reduction in PT pressure, by a factor of 2-5 in [7-12] and even almost 10 in [13, 14]. Also, sometimes plastic straining leads to an appearance of new phases, which were not obtained without plastic straining $[11,15,16]$. Similar results in rotational Bridgman anvils were obtained for various chemical reactions $[17,18]$. Traditionally, PTs under compression in DAC are called "under uniaxial load," while in reality they occur under very heterogeneous multiaxial loading. PTs are characterized by transformation pressure. It depends on the degree of nonhydrostaticity, plastic strain, or plastic shear (in a rotational DAC). This is one of the main reasons (along with different concentration of impurities [19]) why transformation pressure varies in a broad range. For example, PT in zirconium ( $\mathrm{Zr}$ ) from the $\alpha$ phase to the $\omega$ phase under compression in DAC or metallic dies before high pressure torsion (HPT) is reported in the range from 1 to 7 GPa [1924]. The main reason for such discrepancy was the lack of understanding that there is a fundamental difference between pressure-induced PT under hydrostatic conditions and PTs that occur during plastic deformation under high pressure, which are called in $[7,10]$ strain-induced PTs. These two different types of PTs are introduced in $[7,10]$. While pressure-induced PTs start by the nucleation at the pre-existing defects (which serve as pressure and stress tensor concentrators), strain-induced PTs occur by the nucleation at new defects that are continuously generated during the plastic deformation [7, 10]. Dislocations as the main type of defects are generated and densely piled up against grain boundaries or other obstacles during plastic flow, which creates strong concentrators of the stress tensor. The resulting local stresses near stress concentrators may be much higher than the applied pressure and may reach the level required for high pressure phase nucleation. A simple nanoscale analytical model of nucleation at the tip of the dislocation pile-up [7, 10]followed by phase field simulation [25] has demonstrated that 
plastic straining can lead to a substitution of thermally activated nucleation with barrierless nucleation and reduce the PT pressure by a factor of 2-10 or possibly more, similar to experiments in Refs. [7-14]. Strain-induced PTs require a completely different experimental characterization as well as a thermodynamic and kinetic description.

A coarse graining of the above nanoscale theory $[7,10]$ for high-pressure mechanochemistry results in a microscale, strain-controlled (instead of time-controlled), pressure-dependent kinetic equation (see Eq. (S8) in supplemental material) for strain-induced PTs. This kinetic equation represents the main way to characterize strain-induced PTs in experiments. The concept of PT pressure reduces its importance because strain-induced PT can start in quite a broad pressure range. This strain-controlled and pressure-dependent kinetic equation is included in a macroscale model that describes coupled plastic flow and PT with a sample compressed in DAC [26-29] or compressed and twisted in rotational DAC [30-33]. Analytical [7, 10] and finite-element method (FEM) [26-29] have been developed and applied to investigating the evolutions of stresses, strains, and concentration of phases in the entire sample during large plastic flow and PTs under an increasing external loading. This is very important for the interpretation of the experiments because of the strong heterogeneity of all fields, which in most cases is neglected. In experiments, the pressure and concentration of high pressure phases along the radius of a sample at a contact surface has been measured only [34].

In our preceding papers [26-29], FEM was utilized for modeling and simulating the strain-induced PTs under compression in a DAC and interpreting the experimental phenomena. Coupled problems of mechanics and PTs with a large deformation were solved, which involves highly complex equations and FEM simulations. Generic material parameters have been used [26-29] to obtain some generic knowledge of PTs. In this paper, we will study the strain-induced $\mathrm{PT}$ in zirconium $(\mathrm{Zr})$ from the $\alpha$ phase to the $\omega$ phase using specific material parameters from literature. $\mathrm{Zr}$ as a group VI transition metal has widespread applications in the space and aeronautic, biomedical, and nuclear industries. At ambient conditions, the $\alpha$ phase of $\mathrm{Zr}$ possesses the hexagonal closed-packed (hcp) structure. When the pressure is increased at room temperature, martensitic PT into the more open hexagonal structure of $\omega$ phase occurs in the pressure range of 1-7 $\mathrm{GPa}$ [19-24]. With a large plastic shear strain the $\alpha \rightarrow \omega$ PT occurs at around $2 \mathrm{GPa}$ [35], which is considered here as a strain-induced PT, while with hydrostatic 
medium (4:1 ethanol:methanol) PT happens about $7 \mathrm{GPa}$ [23], which is treated here as a pressure-induced PT. Under unconstrained compression before HPT of highly textured $\mathrm{Zr}$ in the direction orthogonal to the c-axes, $\alpha \rightarrow \omega \mathrm{PT}$ starts at $1 \mathrm{GPa}$ [24]. HPT under unconstrained conditions reduces transformation pressure in $\mathrm{Zr}$ down to $0.25 \mathrm{GPa}$ [36]. The main problem is that in Ref. [24] and [36] and in many others pressure was determined as force divided by area, while it is strongly heterogeneous, with much higher pressure at the center than an average pressure. This point will be elaborated in our simulations below. Initiation of the $\alpha \rightarrow \omega$ transformation was observed at intermediate pressures between the above extremes [24, 36]. For example, for nonhydrostatic compression in multianvil system, this PT occurs around $6 \mathrm{GPa}$ [37]. Olinger and Jamieson reported 3.9 GPa as the PT start pressure and suggested that differences in PT start pressure for $\mathrm{Zr}$ in different laboratories are either due to shear stresses or oxygen content [22]. The strong effects of impurities and pressure exposure time on PT pressure for appearance of the $\omega$ phase for different materials are documented in [19]. It is known that the $\omega$ phase of $\mathrm{Zr}$ is preserved under normal pressure [20], which means that there is a considerable hysteresis between $\alpha \rightarrow \omega$ and $\omega \rightarrow \alpha$ transformations [38].

At temperature of $1135 \mathrm{~K}$ and ambient pressure the $\alpha$ phase transforms into the bcc structure ( $\beta$ phase). At room temperature and quasi-hydrostatic loading, the $\omega \rightarrow \beta$ PT has been observed [23] at pressure of about $30 \mathrm{GPa}$. The bcc $\beta$ phase is unstable at the normal pressure and thus the reverse PT takes place upon unloading. However, a recent experiment has successfully stabilized the $\beta$ phase at ambient conditions by using the HPT apparatus, in which five plunger turns were applied under a compression stress of $3 \mathrm{GPa}$ [39]. Later, the $\beta$ phase was obtained by unconstrained HPT and stabilized along with $\omega$ phase after a pressure release at $1 \mathrm{GPa}$ [24] and even 0.25 GPa [36]. As we will discuss in Section 4, these numbers should be increased by a factor of 6 due to the stress heterogeneity.

In this paper, we will present the results of the FEM modeling and simulation of the strain-induced $\alpha \rightarrow \omega$ PT. In Section 2, the problem formulation is presented. In Section 3, the distributions of the components of the stress tenor, pressure, accumulated plastic strain, and concentration of the $\omega$ phase are presented in the volume of a sample at different stages of compression and analyzed. Also, pressure, shear friction stress, the yield strength of two-phase mixture, relative sliding, and normal displacement at the sample diamond boundary are given. 
Changes in sample thickness and maximum pressure are calculated vs. the applied load. In Section 4 the obtained results are used for the interpretation of published experimental data on pressure-, stress-, and strain-induced $\alpha \rightarrow \omega, \alpha \rightarrow \beta$, and $\omega \rightarrow \beta$ PTs in Zr and $\alpha \rightarrow \omega$ PT in Titanium (Ti) under compression and HPT. This includes correction of the reported minimum pressures for these PTs in Zr by a factor of 3 to 6 due stress heterogeneity, analysis of the effect of transmitting media, texture, pressure hysteresis, and reversibility of the PTs. Section 5 contains concluding remarks.

\section{Problem formulation}

In this paper, the shapes of the DAC generally found in experiments (e.g. in $[13,34,40])$ will be used in our FEM models. Typically, at the pressures below $50 \mathrm{GPa}$, a flat bottom contact surface of anvil is used in experiments [13,34], and it will be used it in simulations (see Fig. 1). This is distinct from our previous model that had a bevel angle of $8.5^{\circ}$ for the anvil contact surface under multi-megabar pressures [41]. The sample is initially pre-indented to a thickness of $50 \mu \mathrm{m}$ at $r \leq 150 \mu \mathrm{m}$. We do not use any gasket (similar to [14] and different from [35]), which intensifies the plastic flow by a fast reduction in sample thickness since the yield strength of the $\alpha$ phase is small. Axisymmetric problem formulation is used. Due to symmetry, a quarter of the sample and the anvil will be considered; the geometric parameters can be found in Fig. $1 \mathrm{~b}$ for the anvil and Fig. 1c for the sample. We notice that with an actual sharp angle between lines CD and DE, penetration of the anvil elements into the sample elements may occur. To avoid the divergence in computations due to such a penetration, a smooth transition at point $\mathrm{C}$ is used by a $45^{\circ}$ arc (see Fig. 1c).

The boundary conditions for a quarter of DAC in Fig. $1 \mathrm{~b}$ are summarized as follows:

(1) The normal stress $\sigma_{n}$ is applied at the top of anvil surface.

(2) The radial displacement $u_{r}$ and shear stress $\tau_{r z}$ are zero at the axis $r=0$ (the line AC for the anvil and the line CF for the sample).

(3) At the contact surface CDE, the combined Coulomb and plastic friction model is applied, which will be introduced in detail in the section on friction model.

(4) At the symmetry plane $z=0$ (the plane $\mathrm{FH}$ ), the radial shear stress $\tau_{r z}=0$, and the axial displacement $u_{z}=0$. 
(5) Other surfaces not mentioned above are stress-free.

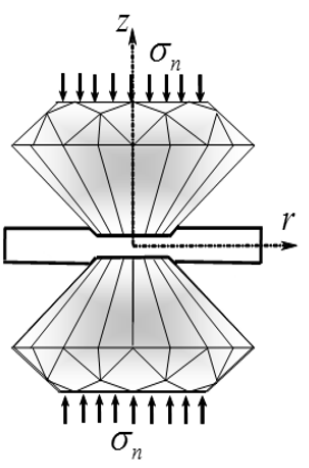

(a)

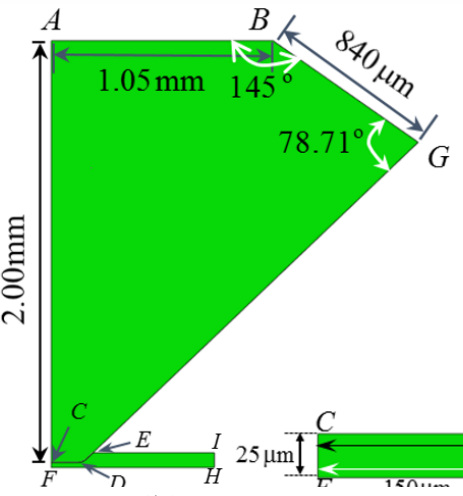

(b)

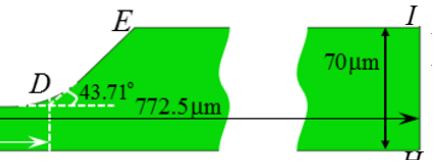

(c)

FIG. 1. (a) Diamond anvil cell scheme, (b) a quarter of the sample and anvil in the initial undeformed state and the geometry of an anvil, and (c) the geometry of a quarter of sample.

Similar to [26-29], the simplest isotropic and perfectly plastic model for the sample is assumed. During the PTs, the changes of elastic properties and yield strength will be considered, whereas in previous models changes in elastic properties were neglected [26-29]. A complete system of equations considering coupled elastoplasticity and strain-induced PTs, and material parameters from experiments and atomistic simulations are introduced in supplemental materials. In addition, the friction model with combination of Coulomb and plastic friction, for contact surfaces between the diamond anvil and the sample, is introduced in supplemental materials.

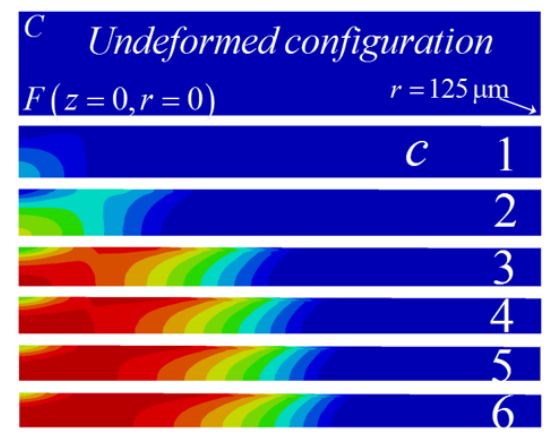

(a)

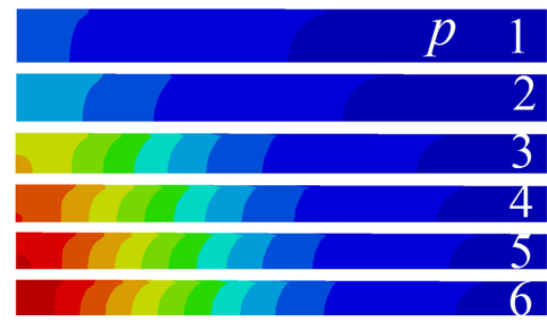

(b)
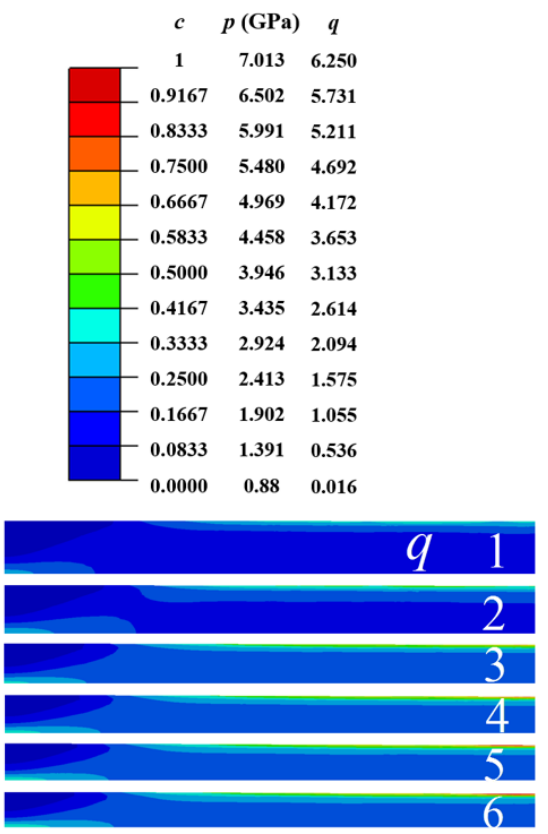

(c) 
FIG. 2 Distributions of concentration $c$ of high pressure phase (a), pressure $p$ (b), and accumulated plastic strain $q$ (c) in the central part of a sample $(0 \leq r \leq 125 \mu \mathrm{m})$, with an increasing applied normal stress $\sigma_{n}$ on the top surface of an anvil. The applied $\sigma_{n}$ is 29.58 MPa (1), 31.92 MPa (2), 38.34 MPa (3), 41.58 MPa (4), 43.74 MPa (5), and 45.42 $\mathrm{MPa}(6)$.

\section{Study of coupled plastic flow and phase transformations}

We will discuss the plastic flows and strain-induced PT in $\mathrm{Zr}$ from the weak $\alpha$ phase to the strong $\omega$ phase at a rising axial compressive load. After a complete PT, the yield strength is increased by 6.56 times; the Young's modulus is increased by $25 \%$; the volume due to the complete PT is reduced by only $1.4 \%$, which is significantly distinct from our previous models with generic parameters [26-29]. Consequently, the results obtained in this paper will be quite different.

Fig. $2 b$ shows that the pressure in a sample continuously reduces from the center to the periphery and that the pressure at the periphery can be much less than the minimum pressure, $p_{\varepsilon}^{d}$, below which a direct strain-induced PT to a high pressure phase cannot occur. Consequently, the nucleation of the high pressure phase does not occur at the periphery, which falls out of our interest; Fig. 2 only shows the fields in the region $(0 \leq r \leq 125 \mu \mathrm{m})$. In the first bar of the undeformed configuration in Fig. 2a, points $C$ and $F$ at the left side correspond to the points $C$ and $F$ in the Fig. 1c, and the sample length in Fig. 2 is $83.3 \%$ of line $C D$ in the Fig. 1c. Due to quite large $p_{\varepsilon}^{d} / \sigma_{y 1}=9.44$, where $\sigma_{y 1}$ is the yield strength of the low pressure phase (vs. $p_{\varepsilon}^{d} / \sigma_{y 1}=6.75$ in the previous studies [26-29]), large thickness reduction occurs before the initiation of the PT at $\sigma_{n}=29.58 \mathrm{MPa}$. Under $\sigma_{n}=29.58 \mathrm{MPa}$, the entire sample plastically deforms with the minimum accumulated strain $q=0.016$ at the contact surface of the sample center and at the major part of the contact surface $q$ is greater than 1 . The PT starts at the center close to the symmetry plane where the pressure reaches $p_{\varepsilon}^{d}=1.7 \mathrm{GPa}$ for the first time, and the PT propagates from the center to the periphery with an increasing load. At the contact surface for $r \leq 10 \mu \mathrm{m}$, there is no displacement along the $r$ direction (stagnation zone, see Fig. 6b), which means that the radial strain $\varepsilon_{r r}$ is almost zero near the contact surface. In addition, the shear stress is also quite small (see Fig. 6a), which leads to a small shear strain as well. That is why the 
accumulated plastic strain is always small at the center close to the contact surface for any normal stress $\sigma_{n}$. Although the shear stress and strain are zero at the symmetry plane, material can freely flow along the $r$ direction there, which causes large radial strain $\varepsilon_{r r}$. With an increasing $\sigma_{n}$, the thickness greatly reduces at the center due to the material radial flow in the region close to the symmetry plane. Consequently, the PT initiates at the center of the sample rather than at the surface, despite the fact that the pressure at the surface is close to that of the symmetry plane. At the periphery of the contact surface, sliding occurs (Fig. 6b) and the radial displacement and its gradient do exist. Because friction stress is equal to the yield strength in shear at the contact surface, the shear flow is pronounced. Consequently, the maximum plastic strain is localized at the periphery of the contact surface since at the symmetry plane there is no shear flow. For $\sigma_{n}=29.58 \mathrm{MPa}$, there is a large plastic strain at the periphery, but the PT does not occurs because of low pressure $\left(p<p_{\varepsilon}^{d}\right)$. At $\sigma_{n}=38.34 \mathrm{MPa}$ and $41.58 \mathrm{MPa}$, the pressure at the contact surface with a large plastic strain reaches and exceeds the minimum pressure $p_{\varepsilon}^{d}$ for the direct PT, which causes a fast nucleation and growth of the $\omega$ phase as seen in Fig. 2a. When the applied stress $\sigma_{n}$ continuously rises (cases (5) and (6) in Fig. 2a), the PT rate is much faster at the contact surface than the rate at the symmetry plane for the same $r$ due to very large plastic strain in the region away from the center. During the compression, thickness reduction causes an intense plastic flow and the thickness of the sample is almost the same along the radial direction due to small elastic deformations of the anvil. Since the thickness reduction at the center is only caused by radial flow in the material close to the symmetry plane, and thickness reduction at the periphery is caused by radial flow throughout the entire thickness, plastic strain at the symmetry plane is larger near the center than at the periphery (Fig. 2c). With an increasing applied normal stress $\sigma_{n}$, the pressure and pressure gradient along the radial direction significantly increase in Fig. 2b. Pressure heterogeneity along the thickness is quite small because of a small length along the sample thickness in comparison with the length along the radius.

Fig. 3 plots the pressure and the concentration $c$ of the $\omega$ phase at the contact surface of the sample. The boundary between the two-phase and the pure $\alpha$ phase zones corresponds to $p=p_{\varepsilon}^{d}$. Since pressure is always larger than $p_{\varepsilon}^{r}=1.2 \mathrm{GPa}$ in the two-phase zone, the reverse 
PT does not occur during loading. Here, $p_{\varepsilon}^{r}$ is the maximum pressure for reverse strain-induced PT, above which reverse PT does not take place (see in supplemental materials). In the singlephase region, the pressure depends linearly on the $r$ coordinate with a small gradient according to simplified equilibrium equation [41, 42]

$$
\frac{d p}{d r}=-2 \tau / h
$$

and friction stress at the contact surface $\tau=\tau_{y}\left(\tau_{y}\right.$ is the yield strength in shear) in the entire contact surface except for the central stagnation region, see Fig. 6a. In the two-phase region, the pressure gradient increases toward the center (excluding central zone), while the pressure gradient decreases in [27] for the case with $\sigma_{y 2}=5 \sigma_{y 1}$, where $\sigma_{y 2}$ is the yield strength of the high pressure phase. In this region, the friction shear stress $\tau$ is equal to the yield strength in shear (see Fig. 6a). The increase of the pressure gradient during the PT according to Eq. (1) is caused by a rising yield strength in shear due to much higher yield strength of the $\omega$ phase. The reduction in the pressure gradient in [27] is due to a large transformational volumetric strain $\bar{\varepsilon}_{t}=-0.1$.

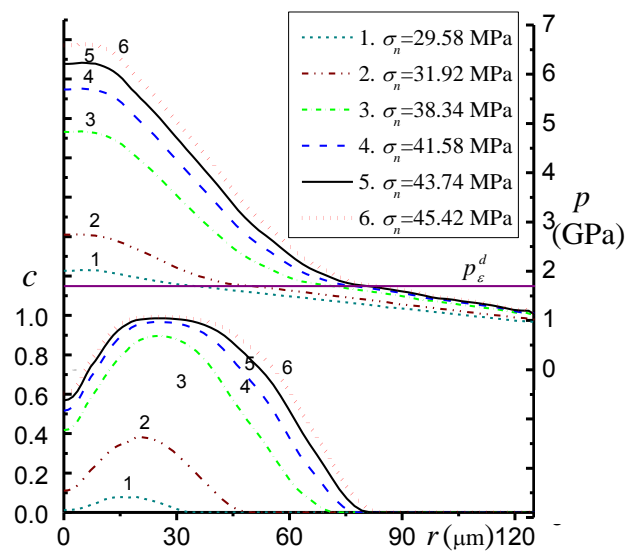

FIG. 3 Distributions of concentration $c$ of high pressure phase and pressure $p$ at the contact surface of sample, with an increasing applied normal stress $\sigma_{n}$.

The evolution of the fields of normal stresses $\sigma_{r r}, \sigma_{z z}$, and $\sigma_{\varphi \varphi}$ in the sample with an increasing applied normal stress $\sigma_{n}$ is shown in Fig. 4. All three normal stresses have close values and distributions because they are larger than the local yield strength. The distributions of the normal stresses $\sigma_{r r}$ and $\sigma_{\varphi \varphi}$ are almost the same for all loads; their magnitudes are slightly smaller than, $\sigma_{z z}$ and their gradients along the thickness are slightly larger than those for $\sigma_{z z}$ in 
the major part of the sample. The gradient $\sigma_{z z}$ in the thickness direction is almost zero, and vertical color boundaries can be found in Fig. 4b. Since all normal stress fields are similar, they are similar to the pressure field in Fig. $2 \mathrm{a}$ as well.

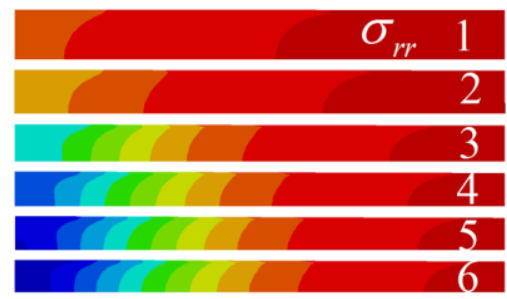

(a)

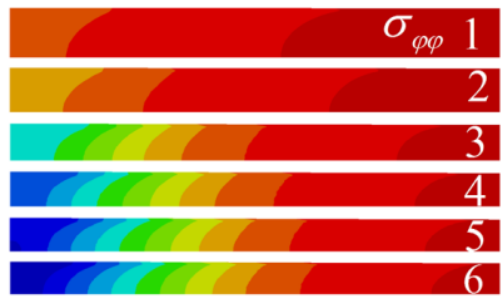

(c)

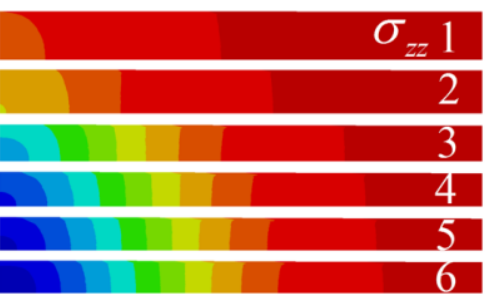

(b)

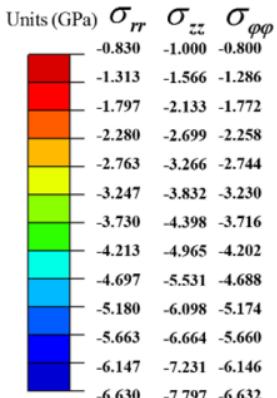

FIG. 4 Distributions of normal stresses $\sigma_{r r}$ (a), $\sigma_{z z}$ (b), and $\sigma_{\varphi \varphi}$ (c) in the sample $(0 \leq r \leq 125 \mu \mathrm{m})$ with an increasing applied normal stress $\sigma_{n}$ at $29.58 \mathrm{MPa}(1), 31.92$ $\mathrm{MPa}$ (2), 38.34 MPa (3), 41.58 MPa (4), 43.74 MPa (5), and 45.42 MPa (6).

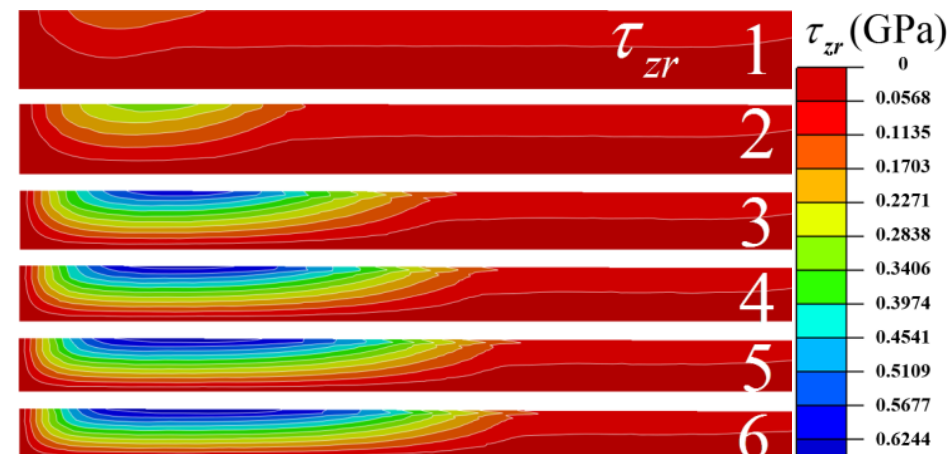

FIG. 5 Distribution of shear stress $\tau_{z r}$ in the sample $(0 \leq r \leq 125 \mu \mathrm{m})$ with an increasing applied normal stress $\sigma_{n}$ at 29.58 $\mathrm{MPa}(1), 31.92 \mathrm{MPa}(2), 38.34 \mathrm{MPa}(3), 41.58 \mathrm{MPa}$ (4), 43.74 $\mathrm{MPa}(5)$, and 45.42 $\mathrm{MPa}(6)$.

Fig. 5 presents the evolution of shear stress $\tau_{z r}$ in the sample with an increasing applied load. Several points govern these fields. (a) The shear stress is zero at the symmetry plane and the $z$ axis. (b) It reaches the local yield strength in shear in the major part of the contact surface (Fig. 6a). (c) Due to significant growth of the yield strength with increasing concentration $c$ of 
the high pressure phase, shear stress at the contact surface grows drastically in the two-phase region away from the symmetry axis. Consequently, the shear stress has a large gradient along the thickness, especially in the two-phase region with maximum concentration $c$.

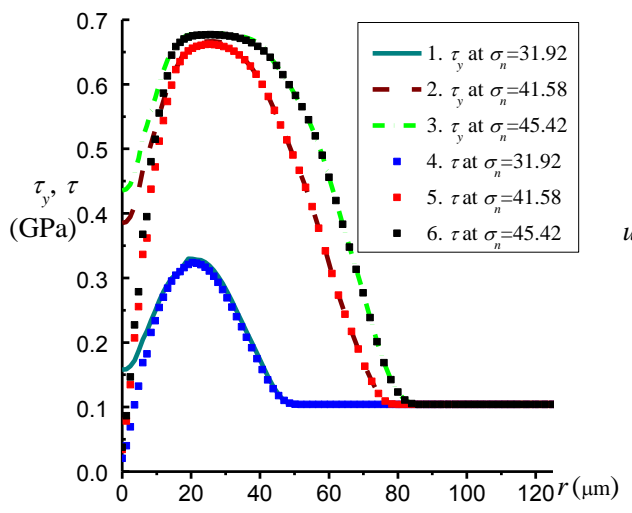

(a)

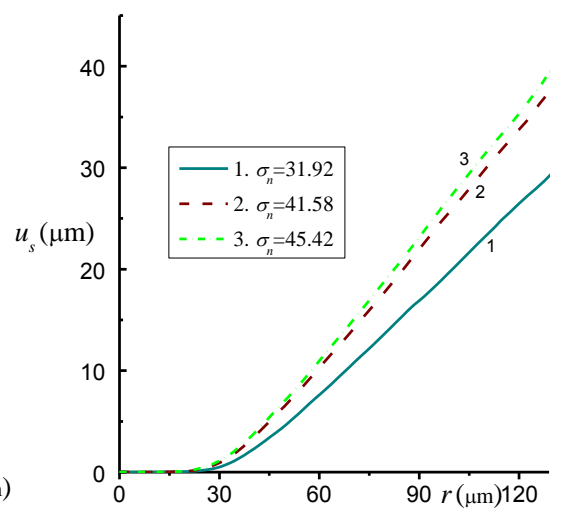

(b)

FIG. 6. (a) Distributions of yield strength in shear $\tau_{y}$ (curves 1,2 and 3) and friction stress $\tau$ (curves 4, 5, and 6) at the contact surface of a sample, and (b) relative slipping distance $u_{s}$ between contact surface of the sample and the anvil.

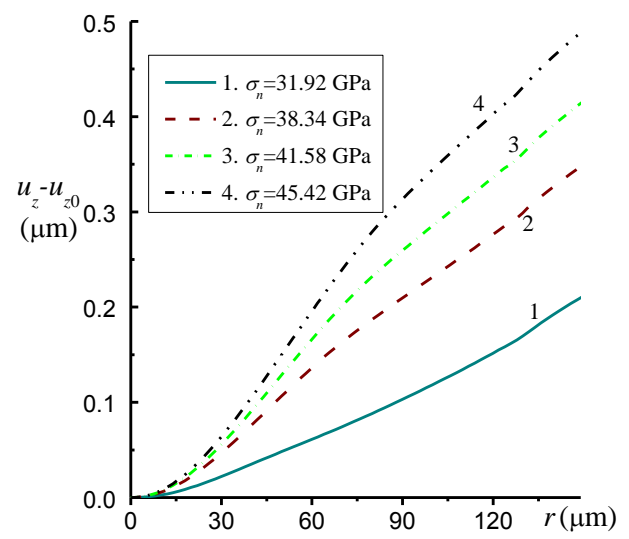

FIG. 7. Relative displacement along the $z$ axis of the points of the anvil contact surface with respect to the point at $r=0$.

The yield strength in shear and friction shear stress are presented under various loads in

Fig. 6a. For the flat surface, $\sigma_{c}=\sigma_{z z}$, and for the almost flat contact surface of current model (see Fig. 5) $\sigma_{c} \approx \sigma_{z z} \approx p$. Since, in the region $r \leq 125 \mu \mathrm{m}, \mu p(\mu=0.3$ is the Coulomb friction coefficient) is larger than the yield strength in shear in Fig. 6a under the applied normal stress $\sigma_{n} \geq 31.92 \mathrm{MPa}$, only the plastic friction operates. The friction stress is equal to the yield strength in shear everywhere except for the central stagnation region where relative sliding is absent (Fig. 6b). Fig. 6b presents the relative slipping distance $u_{s}$ between contact surfaces of the 
sample and the anvil. Below $7 \mathrm{GPa}$ in the sample, the diamond barely deforms. Therefore, the relative slipping distance is close to the displacement of the sample contact surface along the $r$ direction $u_{s} \approx u_{r}$. The results in Figs. $6 \mathrm{a}$ and $6 \mathrm{~b}$ are consistent: there is no sliding when the shear stress is below the yield and where sliding occurs $\tau=\tau_{y}(c)$, where $\tau_{y}(c)$ is the yield strength in shear as a function of the concentration $c$. In the region $15 \mu \mathrm{m}<r<30 \mu \mathrm{m}$, sliding is very small; it significantly and linearly grows for larger radii.

Even under the maximum pressure, $7 \mathrm{GPa}$, the strain in the diamond is quite small: the local maximum normal strain is roughly $0.005=7 \mathrm{GPa} / 1400 \mathrm{GPa}$, where $1400 \mathrm{GPa}$ is Young's modulus. However, the sample is very thin and the radius of sample is quite large so the deformation of diamond anvil cannot be neglected. Fig. 7 shows the relative vertical displacement $u_{z}-u_{z 0}$ between an arbitrary point of the contact surface and at $r=0$, which describes profile of the contact surface. The maximum reduction in the sample thickness at the periphery $(r=150 \mu \mathrm{m})$ due to the deformation diamond is around $1 \mu \mathrm{m}$ in Fig. 7, which is more than $6 \%$ of the sample thickness in Fig. 8 at $\sigma_{n}=45.42 \mathrm{MPa}$. The slope of the relative axis displacement $u_{z}-u_{z 0}$ is variable and it is zero at the $r=0$ due to the symmetry. The bending of the diamond causes a number of interesting phenomena under megabar pressure [41].

The variation of the sample thickness $h$ at $r=0$ and the maximum pressure $p_{\max }$ in the sample is plotted in Fig. 8 versus $\sigma_{n}$. For $\sigma_{n}<5 \mathrm{MPa}$, the plastic region does not cover the entire sample for $r \leq 150 \mu \mathrm{m}$, the pressure gradient is small, and maximum pressure grows slowly with increasing load. For larger $\sigma_{n}$, the pressure gradient increases which causes faster growth of the maximum pressure before reaching $p_{\varepsilon}^{d}$. For $\sigma_{n}<15 \mathrm{MPa}$, the thickness reduction is minor because the plastic region is not completely formed for the outer part of the sample for $r>150 \mu \mathrm{m}$. After the plastic region is fully formed, reduction of the sample thickness increases drastically due to radial flow. Thus, since the $p_{\varepsilon}^{d}$ is 9.44 times the yield strength of $\alpha$ phase, the thickness is reduced to almost half of the initial thickness before the pressure $p_{\max }=p_{\varepsilon}^{d}$ is reached. At the $20 \mathrm{MPa}<\sigma_{n}<35 \mathrm{MPa}$, there is a fast reduction in the thickness because increases of pressure and pressure gradient along the radial direction cause a strong radial material flow. 
After the PT starts for $p_{\max }>p_{\varepsilon}^{d}$, the pressure gradient grows significantly due to the higher yield strength of the $\omega$ phase, which causes much faster growth of $p_{\max }$ with the increase of $\sigma_{n}$. For $\sigma_{n}>35 \mathrm{MPa}$, in addition to the material hardening due to $\mathrm{PT}$, the rate of thickness reduction decreases due to two reasons: (a) For constant sample area and the yield strength, $\sigma_{n}=A+B / h$ with constant $A$ and $B$ according to analytical solution [10], see Eq. (2). Thus, the smaller $h$ is, the smaller rate of its reduction with increasing $\sigma_{n}$. (b) The area of the contact surface grows due to radial flow.

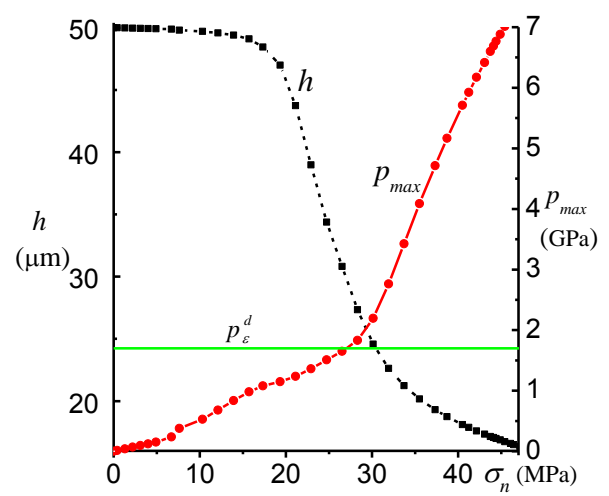

FIG. 8. Variations of the sample thickness $h$ at $r=0$ and the maximum pressure $p_{\max }$ in the sample versus an increasing applied normal stress $\sigma_{n}$.

\section{Discussion}

We will utilize the results obtained here and in $[7,10]$ to interpret some experimentally observed phenomena, including a large scatter in experimental values of transformation pressure. Usually, nucleation of a high pressure phase occurs heterogeneously at some defects (dislocations, grain and twin boundaries), which produce concentrations of all components of the stress tensor. Following [7, 10], we will distinguish three types of PTs.

\section{Pressure-induced phase transformations}

Pressure-induced PTs occur within a liquid medium under a hydrostatic pressure. They start at pre-existing defects. Note that at ambient pressure but under sufficient thermal driving force, thermally-induced PTs start at the same pre-existing defects. The number of pre-existing nucleation defects is limited. That is why for pressure-induced PTs one has to increase pressure to activate less potent defects (i.e., defects with a smaller stress concentrator, e.g., dislocation pile-ups with a smaller number of dislocations). Due to different thermomechanical histories of a sample material during its preparation, the microstructure and distribution of the nucleating 
defects are different for different producers. This explains one source of the spreading of initiation and progress of PTs over the broad pressure range. In addition to the traditional nucleation barrier, which can be overcome by stress concentrations at the defect, the energy of the internal stresses due to transformation strain in high pressure phase may suppress PTs. However, volumetric transformation strain for $\mathrm{Zr}$ is small and this effect can be neglected in the first approximation. An important contribution to the deviation of the pressure from the phase equilibrium pressure for both direct and reverse PTs is an athermal interface friction (dissipation) caused by Peierls barrier, which is an interaction of a moving interface with the stress field of various defects (e.g. point defects, dislocations, grain and twin boundaries and precipitates). Utilizing experimental data for a number of materials in [43] and the theory in [4446], it was suggested in [44-46] that the athermal interface friction and, consequently, pressure hysteresis is proportional to the yield strength $\sigma_{y}(q)$, which is an usually increasing function of the plastic strain $q$ until saturation of the hardening. This seems reasonable because the interface friction characterizes an interaction between a moving interface and the material microstructure, and the yield strength characterizes the resistance to dislocations motion through the same microstructure. Plastic strain dependence of the yield strength and pressure hysteresis explains the second main reason for the significant scatter in PT pressures for both direct and reverse PTs. Samples in different papers could be produced by different methods (rolling, extrusion, or others, often not specified) and have different values of the yield strength, which characterizes microstructure. Depending on the value of strain, the yield strength may change by a factor of 1.5 to 5 for different materials [42]. Thus, for a more complete characterization of the pressureinduced PT one needs to report the yield strength or hardness of a sample and find plastic strain dependence for the yield strength and pressure hysteresis, for $\mathrm{Zr}$ in particular. For unification, it is reasonable to start study with two limiting cases: after long annealing leading to an equilibrium microstructure and after very large plastic deformation below $p_{\varepsilon}^{d}$, to obtain stationary microstructure and saturation of hardening [42].

\section{Stress-induced phase transformations}

Stress-induced PTs occur within nonhydrostatic transmitting media (e.g., after solidification of liquid) or without any transmitting media. Generally, all components of the stress tensor are applied to the sample, but the effective stress does not exceed the macroscopic yield strength. Stress-induced PTs start at the same pre-existing defects as for pressure-induced 
PT. The thermodynamic description of stress-induced PTs is quite similar to that for pressureinduced PTs. Thus, the mechanical part of the thermodynamic driving force, which is work of pressure on the volumetric strain minus change in volumetric elastic energy for the pressureinduced PTs, should be supplemented with the work of deviatoric stresses minus change in the deviatoric part of the elastic energy. In kinematics, the complete transformation deformation gradient that transforms crystal lattice of the parent phase into product phase at zero stresses is taken into account. One more difference is in additional deviatoric strain called transformationinduced plasticity (TRIP). Due to the heterogeneous distribution of the transformation strain during incomplete PTs, they generate internal stresses. When external deviatoric stresses are applied, even below the yield strength but resultant (external and internal) stresses meet the yield criterion, TRIP occurs. Modeling of stress-induced PTs was not performed for high pressure PTs, but is routine, e.g., for shape memory alloys with phenomenological and micromechanical approaches [47] and with phase field nanoscale [48] and microscale [49] approaches. Both of the main sources of the scattering of transformation pressure discussed for pressure-induced PTs, namely, different distributions of nucleating defects and different levels of preliminary plastic strain and consequently an athermal interface friction, due to different treatments of the sample, remain the same for the stress-induced PTs. However, there are some additional sources of a scatter.

To characterize stress-induced PTs, one needs to measure the complete stress tensor, which was never done during PTs under high pressure but was done for shape memory alloys [47]. The main difference between the characterization of PTs under hydrostatic and nonhydrostatic conditions is that under nonhydrostatic conditions stress-strain state is usually quite heterogeneous in the sample. Thus, nonhomogeneous fields should be either measured or calculated in order to determine all parameters in the constitutive equations and characterize PTs. Since neither the magnitude nor distribution of the deviatoric stresses were characterized in experiments, this leads to additional difference in PT pressure in different literature sources. Also, TRIP, similar to plasticity due to applied loads, generates new nucleation sites, produces strain hardening, and increases an athermal friction. Since TRIP depends on external deviatoric stresses, which are not well defined in experiments, this is addition source of a scatter in PT pressure. 
The main problem here is that high pressure is usually produced in the course of plastic compression of sample and gasket. If transmitting media supports the nonhydrostatic stresses, it may produce plastic strain in a sample as well. Thus, it is difficult to distinguish between stressand strain-induced PTs in high pressure experiments.

\section{Strain-induced phase transformations}

If the PTs occur in the course of plastic deformations, they are classified as straininduced PTS. They occur by nucleation at new defects that are permanently generated during plastic deformation. That is why there is no need to increase pressure to promote PTs; PTs can be driven by increasing plastic strains and generating new defects at a constant pressure, and new high pressure phase nuclei will appear near the tips of these defects. Also, stress concentration at the plastic strain-induced defects is usually much stronger than at the pre-existing defects. In particular, the magnitude of all components of the stress tensor at the tip of the dislocation pile up is proportional to the number of dislocations in a pile up [7, 10], and it can be as large as 10 to 100. Thus, one needs a much smaller external pressure to produce the same required PT pressure in a small region near tip of a strong defect. Additional significant driving force appears from the work of deviatoric local stresses and transformation strains, because the magnitude of the deviatoric stresses is not limited by the macroscopic yield strength but by the theoretical strength in shear, which is $G / 10=4.4 \mathrm{GPa}$ for the $\omega$ phase of $\mathrm{Zr}$. This explains the significant reduction of the applied PT pressure in the experiments. That is why the strain-induced PTs require completely different thermodynamic and kinetic treatment and experimental characterization. Followed by three-scale theory in [7, 10], strain-induced PT are characterized by straincontrolled Eq. (S8) in supplemental material, which contains four characteristic pressures.

One of the main points in the interpretation of experiments under compression in DAC without a truly hydrostatic medium is that they occur during plastic deformation and consequently should be treated as strain-induced rather than pressure- or stress-induced. From this point of view, there is no basic difference between the description of the PT in DAC and rotational DAC. The only difference is in the pressure-plastic strain path for each material point of the sample. That is why we modelled PTs in DAC in [26-29] and here as strain-induced, similar to that under shear in rotational DAC [30-33]. The obtained results allow us to interpret possible reasons for the broad range of reported PT pressures in literature. In rotational DAC,

pressure can potentially be constant during PT. In particular, it can be slightly higher than $p_{\varepsilon}^{d}$ 
and these experiments exhibit the strongest reduction in PT pressure due to plastic straining. For compression in DAC, plastic straining occurs during increasing force and consequently pressure, even if it is not required for PT. Because of this, the promoting effect of plastic straining is hidden, see comparison in [33]. Also, pressure increase (in contrast to plastic strain increase) is not a basic characteristic of a PT; this is just a result of system reaction on deformation.

\section{Correction to reported transformation pressures for compression and high pressure torsion}

Compression of material before HPT and deformation during HPT, under unconstrained conditions, are very similar to compression in DAC and deformation in a rotational DAC. For HPT, the reported PT pressure is equal to the applied force divided by area, which is only true for homogeneous pressure distribution. It follows from Figs. 2-4 that pressure and all normal stresses are very heterogeneous along the radius, and a similar distribution is valid for sample compression before HPT because the thickness of the sample significantly reduced [24]. Without PTs, FEM results can be well described (see comparison in [50]) with known solution of the simplified equilibrium equation (1). After a large enough reduction in the thickness the contact friction shear stress $\tau$ reaches its maximum possible value $\tau_{y}=\sigma_{y} / \sqrt{3}$ (von Mises yield condition is used; for Tresca yield condition $\left.\tau_{y}=\sigma_{y} / 2\right)$. The solution to this equilibrium equation is

$$
p=\sigma_{0 r}+\sigma_{y}[1+2(R-r) / \sqrt{3} h] ; \quad P=\sigma_{0 r}+\sigma_{y}[1+2 R / 3 \sqrt{3} h],
$$

where $\sigma_{0 r}$ is the radial normal stress at the edge $r=R$ ( $R$ is the radius of diamond cullet (the flat bottom part)), and $P$ is the averaged pressure obtained as the force calculated for the pressure distribution in the first Eq. (2) per unit area. For unconstrained HPT $\sigma_{0 r}=0$. If $P>>\sigma_{y}$, which is the case for a large $R / h$, then the term $\sigma_{y}$ in Eq.(2) can be neglected. Eq. (2) reduces to the following expressions for the maximum pressure $p_{\max }$ at the center of a sample $r=0$ and the averaged pressure $P$ :

$$
p_{\max } \approx 2 \sigma_{y} R / \sqrt{3} h ; \quad P \approx 2 \sigma_{y} R / 3 h \sqrt{3}, \quad \text { and } \quad p_{\max }=3 P .
$$

Thus, reported averaged pressures for PTs under unconstrained compression should be multiplied by a factor of approximately 3 because PT starts close to the center where pressure is equal to $p_{\max }$. In particular, the lowest reported transformation pressure under unconstrained 
compression before HPT of highly textured $\mathrm{Zr}$ was reported at $1 \mathrm{GPa}$ [24]. Based on Eq. (2), it should be about $3 \mathrm{GPa}$.

Note that for analytical solution for conditions similar to the HPT under a constant force, without PT, the thickness of the sample reduces but pressure distribution does not change[7, 10]. This corresponds to experiments [11,43] and FEM solutions [50]. Thus, the minimum pressure for $\alpha \rightarrow \omega$ PT under large torsion, $0.25 \mathrm{GPa}$ (in [36]) should be corrected to $0.75 \mathrm{GPa}$. This value can be considered as $p_{\varepsilon}^{d}$ for commercially pure $\mathrm{Zr}$ in [36].

\section{Comments on strain-induced $\alpha \rightarrow \beta$ and $\omega \rightarrow \beta$ phase transformations}

Note that the $\alpha \rightarrow \beta$ (or $\omega \rightarrow \beta$ ) PT was also obtained along with $\alpha \rightarrow \omega$ PT under compression before torsion at the averaged pressure of $1 \mathrm{GPa}$ in [24] and under 5 turns of torsion at averaged pressure of $0.5 \mathrm{GPa}$ in [36]. This is amazing because under quasi-hydrostatic conditions this transformation occurs above $30 \mathrm{GPa}$ [51]. These numbers should be also corrected by a factor of at least 3 if the PT to $\beta$ occurs in a sample consisting of pure $\alpha$ phase or $\omega$ phase. However, when the central part of the sample has transformed to the $\omega$ phase, like in Figs. 2 and 3, significant local pressure growth is observed under compression; even stronger pressure growth takes place under torsion under a constant force (see [31]). The reason is in the much higher yield strength of the $\omega$ phase than the $\alpha$ phase. Since pressure increases in the central region only, it does not contribute significantly to the average pressure [7, 10]. Each case should be considered separately, but based on Fig. 3, pressure at the center could be two times larger than interpolated to the center pressure for the pure $\alpha$ phase. Thus, the resultant corrections $[24,36]$ should be multiplied by a factor of 6 , i.e., strain-induced $\alpha \rightarrow \beta$ (or $\omega \rightarrow \beta$ )

PT occurs at $6 \mathrm{GPa}$ at compression and at $3 \mathrm{GPa}$ at HPT. The last value can be interpreted as $p_{\varepsilon}^{d}$, which is an order of magnitude smaller than the PT pressure under quasi-hydrostatic conditions. Note that an order of magnitude reduction in the pressure for strain-induced PT in comparison with PT under quasi-hydrostatic conditions was reported for PT from rhombohedral to cubic BN under compression in [14] and for PT from hexagonal to wurtzitic BN under compression and torsion in rotational DAC in [13]. Note that the pressure in [13, 14] was in-situ measured locally. The same corrections are applicable to the PT in $\mathrm{Zr}-\mathrm{Nb}$ alloys with even larger ratios of pressures for pressure-induced and strain-induced PTs [36]. 
One more factor may promote appearance of the $\beta$ phase, which is the heterogeneity of stress and strain in a two-phase $\alpha+\omega$ mixture due to a significantly larger strength of the $\omega$ phase. In particular, plastic strain will be significantly larger for the $\alpha$ phase, which promotes its transformation. Due to larger strength, the $\omega$ phase may have larger stresses (pressure), which will promote $\omega \rightarrow \beta$ PT. Depending on the morphology of the mixture, various stress-strain conditions are possible. In particular, the contact of two $\omega$ phase particles along small area may produce a large stress concentration and $\omega \rightarrow \beta$ PT. If there is a thin interlayer of the weak $\alpha$ phase between two grains, it is similar to the compression of a thin sample by rigid anvils, like in Section 3, which will cause a large stress gradient and stresses much larger than the average both in the $\alpha$ phase interlayer and $\omega$ phase grains. Still, it is not clear which of PTs, $\alpha \rightarrow \beta$ or $\omega \rightarrow \beta$ or both simultaneously occur. According to Usikov-Zilbershtein crystallographic mechanism [52] , $\alpha \rightarrow \omega$ PTs in $\mathrm{Zr}$ and $\mathrm{Ti}$ occur through intermediate thermodynamically unstable $\beta$ phase. This mechanism was not considered realistic because $\beta$ phase was not previously observed in experiments under hydrostatic and quasi-hydrostatic conditions. However, intermediate unstable phase should not, in general, be observed especially after unloading to the ambient pressure, unless it is somehow stabilized. Also, the $\beta$ phase was observed in Ti-(10 at. $\%$ V) after high pressure treatment [19]. Recently, the $\beta$ phase was found along with $\omega$ phase after plastic compression before HPT at $1 \mathrm{GPa}$ [24] and after HPT at $0.5 \mathrm{GPa}$ [36]. In this case, if it appears as an intermediate phase, it was arrested by large plastic deformation. Alternatively, plastic deformation can change the mechanism of the PT and transformation path. It is known that, [43] e.g., for PTs in $\mathrm{Ge}$ and $\mathrm{Si}$, under hydrostatic conditions phase I transforms to phase II and under plastic straining PT occurs along the path $\mathrm{I} \rightarrow \mathrm{III} \rightarrow \mathrm{II}$. Also, the intermediate $\beta$ phase may appear at the interface between $\alpha$ and $\omega$ phases and it can be stabilized by reduction in the total interfacial energy. Similar PTs via intermediate phases were discussed in [53] and [54] and described by phase field approach [55].

\section{Orientation dependence of the $\alpha \rightarrow \omega$ PT under compression before torsion}

Let us further analyze the results in [24] under compression before HPT for textured Zr. The PT was detected during compression to $1 \mathrm{GPa}$ orthogonal to the c-axes, which had a hardness of $107 \mathrm{Hv}$, but it was not obtained during a similar compression in the direction parallel to the c-axes, which exhibited a hardness of $129 \mathrm{Hv}$. In fact, the authors of [24] used our 
nanoscale theory [10] for interpretation of their results. Namely, while compression in the direction parallel to the c-axes occurs solely by the dislocation mechanism along pyramidal slip

systems, compression in the direction orthogonal to the c-axes involves $\{10 \overline{1} 2\}$ twinning in addition to prismatic slip. Twin boundaries produce additional obstacles for dislocation pile-ups and additional nucleation cites. Also, it was stated that due to a lower yield strength in the orthogonal direction, plastic strain was larger and it produced stronger defects, which caused nucleation. Indeed, reduction in a sample thickness for compression in the orthogonal direction $(48 \%)$ is significantly larger than the $30 \%$ in the parallel direction. However, pressure heterogeneity is not considered in [24] and we believe that it plays the major role.

Pressure gradient is proportional to frictional stress at the contact surface $\tau$ (Eq. (1)). Friction stress $\tau$ increases during compression until it reaches the yield strength in shear; it is larger for a larger thickness reduction and probably reached everywhere $\tau_{y}$ for compression in the perpendicular direction but did not for compression in the parallel direction. Since the pressure gradient is also inversely proportion to the thickness $h$, a larger reduction in thickness for perpendicular direction leads to a larger pressure gradient. The larger pressure gradient leads to larger pressure at the center, accelerating PT kinetics according to Eq. (S8). Also, for compression parallel to the c-axes direction, $\sigma_{0 r}$ is not zero and $\sigma_{y}$ is large. This is why a factor connecting $p_{\max }$ and $P$ is smaller. During PTs, pressure further grows near the center due to larger yield strength of the $\omega$ phase, which leads to positive mechanochemical feedback and growing concentration of the high pressure phase.

\section{Effect of transmitting media on $\alpha \rightarrow \omega$ transformation in Ti}

The systematic study of the effect of transmitting media on the $\alpha \rightarrow \omega \mathrm{PT}$ in Ti, which is very close to $\alpha \rightarrow \omega \mathrm{PT}$ in $\mathrm{Zr}$, was presented in [56]. Let us interpret these results within the framework discussed here. The PT within argon medium can be considered pressure-induced, at least below $13 \mathrm{GPa}$, and it starts at $10.5 \mathrm{GPa}$ and completes at $14.9 \mathrm{GPa}$. In 4:1 methanolethanol medium, the $\alpha \rightarrow \omega$ PT occurs in the range $10.2-14.7 \mathrm{GPa}$. This small and the same reduction in PT start and finish pressures, as well as pressure for any intermediate concentration of the $\omega$ phase can be rationalized in the increase of the thermodynamic driving force due to work of nonhydrostatic (deviatoric) stresses and transformation strains. This increase cannot be large because of the relatively low yield strength of the $\alpha$ phase of 0.3-0.6 GPa in [57] vs. 
applied pressure on the order of $10 \mathrm{GPa}$. This is a typical stress stress-induced PT under high pressure. For both cases the stress state of a sample should be quite homogeneous. After pressure release, the $\omega$ phase retains. This is logical, because if we assume a phase equilibrium pressure at $2 \mathrm{GPa}$ in [19], pressure deviation for a direct phase transition is at $8 \mathrm{GPa}$; similar deviation for the reverse PT would require negative pressures. Also, due to the homogeneity of stress and strain fields, chances for untransformed $\alpha$ phase regions, which could serve the embryos for reverse PT, are not high.

PTs in $\mathrm{NaCl}$ medium and without a transmitting medium occurred in the range 6.2-14.2 GPa and 4.9-12.4 GPa, respectively. The pressure was measured by ruby crystal, and there is no description about the location of measurements. It is also unknown where the concentration of the $\omega$ phase was measured. In any case, without transmitting media, plastic deformation of $\mathrm{Ti}$ occurs and the PT is strain-induced. This explains significant reduction of the PT pressure, as it was discussed above. Since pressure - concentration of the $\omega$ phase curve for the PT within $\mathrm{NaCl}$ looks like that of the compression without transmitting medium, but shifted up by $\sim 1.5$ $\mathrm{GPa}$ and is significantly lower than for quasi-hydrostatic conditions, this transformation most likely occurs during plastic deformation of a sample and is strain-induced. Note that the heterogeneity of all fields for the compression of a sample within strong gasket (like 301 stainless steel or rhenium) is much smaller than for the compression without a gasket in Section 3 , see solutions in [29].

The above discussion is consistent with one more experimental result [56] that the smaller PT pressure is due to deviatoric stresses and plastic strain the larger the full-width at half-maximum of the diffraction peak values is. Indeed, this width characterizes the stresses heterogeneity and deviatoric stresses.

Another very interesting observation in [56] is that after the PT in Ti within argon and methanol-ethanol medium, there is no reverse PT; however, after the PT within $\mathrm{NaCl}$ and without transmitting medium, significant concentrations of the $\alpha$ phase appears at a quite high pressure. If we consider that the reverse PT is pressure- or stress-induced, these results look contradictory. As it was discussed in [7, 10, 43-46] and above, PT hysteresis is proportional to the yield strength, which should be larger after strain-induced PT due to strain hardening. Our hypothesis is that after the strain-induced $\mathrm{PT}$ within $\mathrm{NaCl}$ and without any medium, plastic strain occurs in the sample during unloading, which causes reverse PT. Such a result was obtained in 
[28], where the same model for a strain-induced PT was applied to the simulation of unloading for sample without gasket. With a strong gasket, plastic straining of the sample during unloading may be even more pronounced. Indeed, because Young's modulus of steel is significantly larger than for $\mathrm{Ti}$ and $\mathrm{NaCl}(40 \mathrm{GPa})$, elastic strain in steel is significantly smaller, and stress in a gasket reduces during unloading faster than in a sample. That is why during unloading the difference between axial and radial stresses in a sample exceeds the yield strength of the $\mathrm{Ti}$ and plastic deformation occurs, which causes the strain-induced reverse PT. Transformation strain during this PT under the nonhydrostatic condition causes TRIP and additional strong defects leading to reverse PT. In addition, stress heterogeneity along the radius may result in pressures lower than the reported in [56]. At the nanoscale, a dislocation pile up that appeared during plastic flow and causing $\alpha \rightarrow \omega \mathrm{PT}$, possesses at its tip compressive and tensile stresses of the same magnitude but in different regions. Tensile stresses either cause reverse PT in the $\omega$ phase, or retain small regions of the $\alpha$ phase that can serve as preexisting nuclei for $\omega \rightarrow \alpha$ PT under pressure release. That is why the kinetic Eq. (S8) included both direct and reverse PTs at the same time. Also, residual stresses caused by transformation strain during direct PT promote reverse lattice transformation during reverse PTs. For pressure- and stress-induced PTs, preexisting defects have much lower stress concentration and much smaller promotion of both direct and reverse PTs.

It was known in literature that shear stresses reduce PT hysteresis; it was deduced from here that if they reduce hysteresis to zero (e.g., for $\alpha \leftrightarrow \omega \mathrm{PT}$ in $\mathrm{Zn}$ and Ti [35] and B1 $\leftrightarrow \mathrm{B} 2$ $\mathrm{PT}$ in $\mathrm{KCl}$ [43] ), then PT pressure is the phase equilibrium pressure. However, for some PTs (e.g., semiconductor-metal in InTe, InSb, Si I $\rightarrow$ II, and Ge I $\rightarrow$ II [43] ), the pressure for direct PTs under plastic shear was lower than the phase equilibrium pressure. The theory [7, 10] explained such a strong pressure reduction for strain-induced PT and predicted that description of strain-induced PT at the microscale does not include the phase equilibrium pressure, see Eq. (S8).

The final interesting results in [56] is that at $5 \mathrm{GPa}$ Ti was heated to the region of stability of the high-temperature $\beta$ phase, $1750 \mathrm{~K}$, or melt, $2150 \mathrm{~K}$, and quenched. After quenching, a $\alpha+\omega$ mixture was observed, i.e., $\omega$ phase was obtained under pressure of $5 \mathrm{GPa}$, at which it was not obtained under cold compression. This result was explained by thermal fluctuations at high temperatures that could promote nucleation at lower pressure. We can add that both the 
yield strength and pressure hysteresis, which is proportional to the yield strength, reduce with increasing temperature which leads to a reduction in transformation pressure with temperature, while the equilibrium pressure grows. Finally, the low-pressure metastable continuation of the $\beta-\omega$ equilibrium line in pressure-temperature plane is below the $\beta-\alpha$ equilibrium line [51]. If some $\beta$ crystals did not transform to $\alpha$ during quenching, then after the temperature reduces below the metastable continuation of the $\beta-\omega$ equilibrium line, the $\beta \rightarrow \omega$ PT is possible as well and it kinetically competes with the $\beta \rightarrow \alpha$ PT. Similar situations with metastable continuation of the equilibrium lines was reported in $[53,58,59]$ for PTs in ice, $\mathrm{Si}, \mathrm{Ge}$, and other materials.

\section{Concluding remarks}

In this paper, the plastic flows and the strain-induced $\alpha \rightarrow \omega \mathrm{PT}$ in $\mathrm{Zr}$ under compression in the sample are investigated using FEM. Very heterogeneous fields of stress tensor, accumulated plastic strain, and concentration of the $\omega$ phase are obtained during the applied load increase. The sample thickness reduces by $50 \%$ from the initial one before the PT starts, which is because of a large ratio $p_{\varepsilon}^{d} / \sigma_{y 1}$. The PT starts in the region close to the symmetry plane at the center of sample when pressure exceeds the minimum pressure $p_{\varepsilon}^{d}=1.7 \mathrm{GPa}$, and it propagates from the center to the periphery. The maximum plastic strain is localized at the contact surface and is larger toward the periphery; once the pressure exceeds $p_{\varepsilon}^{d}$ at the periphery of the contact surface, the PT rate is faster at the surface than at the symmetry plane. With an increasing applied normal stress $\sigma_{n}$, the pressure gradient along the radial direction significantly increases, especially in two-phase region, which is due to a rising yield strength in shear during PT and a reduction in sample thickness. The distributions of the normal stresses $\sigma_{r r}$ and $\sigma_{\varphi \varphi}$ are almost the same under different loads, and the gradient of $\sigma_{z z}$ along the thickness is almost zero. All three normal stresses $\sigma_{r r}, \sigma_{z z}$ and $\sigma_{\varphi \varphi}$ as well as pressure $p$ have close values and distributions because of relatively low yield strength. The shear stress is zero at the symmetry plane and it grows to the maximum value at the contact surface equal to the yield strength in shear $\tau_{y}(c)$ in the major part of the contact surface. Consequently, the shear stress has a large gradient along the thickness, especially in the region with a high concentration of the high pressure phase $c$. In the 
major part of the contact surface, the plastic friction (instead of Coulomb friction) operates. The region with the maximum concentration of the $\omega$ phase is shifted from the region with maximum pressure to the region with larger plastic strain. Even at a maximum pressure of $7 \mathrm{GPa}$ the PT was not completed everywhere. This does not mean that this strain-induced PT cannot be completed below $7 \mathrm{GPa}$; it can if, for example, pressure slightly above $p_{\varepsilon}^{d}=1.7 \mathrm{GPa}$ will be kept and rotation of an anvil will be applied. Here, pressure grows during compression and PT, and this growth depends on the initial geometry and the yield strength of phases. With an increasing load, the pressure and pressure gradient along the radial direction significantly increase in twophase region due to much larger yield strength of the $\omega$ phase. This, in turn promotes PT, which produces positive mechanochemical feedback.

Under the maximum pressure of $7 \mathrm{GPa}$, the strain in the diamond is quite small. However, the deformation of the diamond anvil cannot be neglected due to a large length in the radial direction. The thickness reduction at the sample periphery due to the deformation diamond is around $1 \mu \mathrm{m}$ which is more than $6 \%$ of the sample thickness. With an increasing applied normal stress $\sigma_{n}$, the of thickness reduction passes through three stages: (1) very small reduction until the plastic zone covers the entire sample between anvils; (2) sharp increase in thickness due to large radial pressure gradient material flow; (3) reduction in the rate of thickening because of the analytical solution Eq. (2), growth of the area of the contact surface, and increase in the yield strength $\tau_{y}(c)$ during the PT.

Various experimental results have been interpreted. The simplified analytical solution shows that the maximum pressure at the center of a sample is three times larger than an averaged pressure. This is true both for plastic compression and torsion under fixed load without PT. Since the averaged pressure is used for characterizing PT under HPT, but PT starts in the region with maximum pressure, all published PT pressure for HPT should be multiplied by a factor of 3. Thus, the minimum pressure for $\alpha \rightarrow \omega$ PT under large torsion, $0.25 \mathrm{GPa}$ in [36] should be corrected to $0.75 \mathrm{GPa}$, which can be considered as $p_{\varepsilon}^{d}$ for commercially pure Zr. Even larger corrections should be applied to interpret very low pressure for $\alpha \rightarrow \beta$ or $\omega \rightarrow \beta$ PT, which occurs along with $\alpha \rightarrow \omega$ PT under compression at averaged pressure of $1 \mathrm{GPa}$ in [24] and under HPT at $0.5 \mathrm{GPa}$ in [36] (instead of $30 \mathrm{GPa}$ under quasi-hydrostatic conditions [51]). In addition to a factor of 3 , which is applied for single phase material, significant local pressure 
growth is observed at the center during $\alpha \rightarrow \omega$ PT due to higher yield strength of the $\omega$ phase. Based on our simulations, it is suggested to additionally multiply and averaged pressure by a factor of 2 , i.e., by a total factor of 6 . This leads for $p_{\varepsilon}^{d}=3 \mathrm{GPa}$ for appearance of the $\omega$ phase. Also, local stresses within two-phase $\alpha+\omega$ mixture may significantly deviate from the averaged stresses.

One of the main reasons for different laboratories reporting different pressures is that depending on the transmitting media, PTs can be pressure-induced (under hydrostatic conditions), stress-induced (under nonhydrostatic stresses below the yielding), and strain-induced during plastic deformation. Since in most cases a sample deforms plastically during an increase in pressure, PTs are strain-induced. Strain-induced PTs require completely different thermodynamic and kinetic treatment and experimental characterization than the pressure- and stress-induced PT, e.g., in terms of strain-controlled kinetic equation of the type of Eq. (S8). In particular, plastic strain promotes strain-induced PTs; however, plastic strain before a PT suppresses the PT because it increases the athermal interface friction, which is proportional to the yield strength $\sigma_{y}(q)$. That is why different thermomechanical histories of a sample material during its preparation, microstructure, $\sigma_{y}(q)$, and distribution of the nucleating defects are different for different producers and laboratories, which results in a large scatter in PT pressures. With the reduction of the degree of nonhydrostaticity of the transmitting medium, transition from pressure- to stress- to strain-induced PT occurs, leading to different degrees of reduction in PT pressure.

While pressure- and stress-induced $\alpha \rightarrow \omega$ PTs within argon and methanol-ethanol medium were irreversible, strain-induced $\alpha \rightarrow \omega$ PTs within $\mathrm{NaCl}$ and without transmitting medium were partially reversible [56]. This was explained by the presence of the plastic strain under unloading due to stress heterogeneity for strain-induced PTs, which causes reverse straininduced PT at high pressure $p_{\varepsilon}^{r}$. At the nanoscale, a dislocation pile up that appeared during plastic flow and caused $\alpha \rightarrow \omega$ PT, possesses at its tip compressive and tensile stresses of the same magnitude but in different regions. Tensile stresses either cause reverse PT in the $\omega$ phase or retain small regions of the $\alpha$ phase that can serve as preexisting nuclei for $\omega \rightarrow \alpha$ PT under pressure release. Also, quenching the high-temperature $\beta$ phase at $5 \mathrm{GPa}$ lead to $\alpha+\omega$ mixture, while the $\omega$ phase could not be obtained at $5 \mathrm{GPa}$ under hydrostatic pressure [56]. This was 
explained by a reduction of the yield strength and pressure hysteresis at high temperature and also by crossing low-pressure metastable continuation of the $\beta-\omega$ equilibrium line.

As the next step, PTs among three phases of $\mathrm{Zr}$ will be considered under compression in DAC, shear in rotational DAC or HPT [30-33]. High pressure PTs in Zr under high-strain-rate loading are also of great interest for both experiments and theories (e.g. [60-62]). These as well as shock induced PTs in other materials, e.g., in Tantalum [63] can be studied using the same model complemented with strain-rate dependence.

\section{Acknowledgements}

The support of NSF (DMR-1434613), ARO (W911NF-12-1-0340), DARPA (W31P4Q-13-10010), and Iowa State University (Schafer 2050 Challenge Professorship) is gratefully acknowledged.

\section{References}

[1] L, Dubrovinsky, N. Dubrovinskaia, S. Saxena, T. LiBehan, X-ray diffraction under non-hydrostatic conditions in experiments with diamond anvil cell: wustite (FeO) as an example, Mat. Sci. Eng. A. 288 (2000) 187-190.

[2] C. Nisr, G. Ribarik, T. Ungar, G. B. M. Vaughan, P. Cordier, S. Merkel, High resolution three-dimensional X-ray diffraction study of dislocations in grains of $\mathrm{MgGeO} 3$ post-perovskite at $90 \mathrm{GPa}$, J. Geophys. Res. 117 (2012) B03201.

[3] A. R. Oganov, J. H. Chen, C. Gatti, et al., Ionic high-pressure form of elemental boron, Nature 457 (2009) $863-867$.

[4] C. S. Zha, Z. X. Liu, R. J. Hemley, Synchrotron infrared measurements of dense hydrogen to 360 GPa, Phys. Rev. Lett. 108 (2012) 146402.

[5] P. W. Bridgman. Effects of High Shearing Stress Combined with High Hydrostatic Pressure. Phys. Rev. 48 (1935) 825847.

[6] K. Edalati and Z. Horita. A review on high-pressure torsion (HPT) from 1935 to 1988, Mat. Sci. Eng. A. 652 (2016) 325-352.

[7] V. I. Levitas, "Continuum mechanical fundamentals of mechanochemistry," in High-pressure surface science and engineering, edited by Y. Gogotsi and V. Domnich, Institute of Physics Publishing, 2004.

[8] M. M. Alexandrova, V. D. Blank, S. G. Buga, Phase transitions in Ge and Si under shear strain under pressure up to 12 GPa and p-t- $\gamma$ diagrams of these elements, Solid State Phys. 35 (1993) 1308-1317.

[9] V. D. Blank, Y. Y. Boguslavsky, M. I. Eremets, E. S. Itskevich, Y. S. Konyaev, A. M. Shirokov, E. I. Estrin, Pressure Self-Multiplication Effect on Phase-Transition under Quasi-Hydrostatic Conditions, Zh. Eksp. Teor. Fiz. 87 (1984) 922-926.

[10] V. I. Levitas, High-pressure mechanochemistry: Conceptual multiscale theory and interpretation of experiments, Phys. Rev. B 70 (2004) 184118.

[11] N. V. Novikov, S. B. Polotnyak, L. K. Shvedov, V. I. Levitas, Synthesis, Sintering and properties of Superhard Materials, Superhard Mater. 3 (1999) 39-51.

[12] V. D. Blank, Z. H. Malyushitska, B. A. Kulnitskiy, Structural Mechanisms of Si (Ge) I $\rightarrow$ III Phase Transformations, High Press. Phys. Eng. 3 (1993) 28.

[13] C. Ji, V. I. Levitas, H. Y. Zhu, J. Chaudhuri, A. Marathe, Y. Z. Ma, Shear-induced phase transition of nanocrystalline hexagonal boron nitride to wurtzitic structure at room temperature and lower pressure. P. Natl. Acad. Sci. USA 109 (2012) 19108-19112.

[14] V. I. Levitas, L. K. Shvedov, Low-pressure phase transformation from rhombohedral to cubic BN: Experiment and theory, Phys. Rev. B 65 (2002) 104109.

[15] V. D. Blank, S. G. Buga, Automated optical installation with a shear diamond-anvil cell, Instrum. Exp. Tech. 36 (1993) 149-157. 
[16] V. I. Levitas, Y. Z. Ma, E. Selvi, J. Z. Wu, J. A. Patten, High-density amorphous phase of silicon carbide obtained under large plastic shear and high pressure, Phys. Rev. B 85 (2012) 054114.

[17] E. Teller. On the Speed of Reactions at High Pressures. J. Chem. Phys. 36 (1962) 901.

[18] A. A. Zharov, "Reaction of Solid Monomers and Polymers under Shear Deformation and High Pressure." in High Pressure Chemistry and Physics of Polymers, ed by A L Kovarskii, CRC Press, 1994.

[19] S. K. Sikka, Y. K. Vohra, R. Chidambaram, Omega phase in materials, Prog. Mater. Sci., (1982), 27, $245-310$.

[20] J. C. Jamieson, Crystal structures of titanium, zirconium, and hafnium at high pressures, Science 140 (1963) $72-73$.

[21] A. Jayaraman, W. Klement, G. C. Kennedy, Phase Diagrams of Arsenic, Antimony, and Bismuth at Pressures up to 70 kbars, Phys. Rev. 131 (1963) 632-637.

[22] B. Olinger, J. C. Jamieson, Zirconium: Phases and compressibility to 120 kilobars, High Temp. High Press. 5 (1973) 123-131.

[23] H. Xia, S. J. Duclos, A. L. Ruoff, Y. K. Vohra, New High-Pressure Phase-Transition in Zirconium Metal, Phys. Rev. Lett. 64 (1990) 204-207.

[24] B. Srinivasarao, A. P. Zhilyaev, M. T. Perez-Prado, Orientation dependency of the alpha to omega plus beta transformation in commercially pure zirconium by high-pressure torsion, Scripta Mater. 65 (2011) 241-244.

[25] V. I. Levitas, M. Javanbakht, Phase transformations in nanograin materials under high pressure and plastic shear: nanoscale mechanisms, Nanoscale 6 (2014) 162-166.

[26] V. I. Levitas, O. M. Zarechnyy, Modeling and simulation of strain-induced phase transformations under compression in a diamond anvil cell, Phys. Rev. B 82 (2010) 174123.

[27] B. Feng, V. I. Levitas, O. M. Zarechnyy, Plastic flows and phase transformations in materials under compression in diamond anvil cell: Effect of contact sliding, J. Appl. Phys. 114 (2013) 043506.

[28] B. Feng, O. M. Zarechnyy, V. I. Levitas, Strain-induced phase transformations under compression, unloading, and reloading in a diamond anvil cell, J. Appl. Phys. 113 (2013) 173514.

[29] B. Feng, V. I. Levitas, Y. Z. Ma, Strain-induced phase transformation under compression in a diamond anvil cell: Simulations of a sample and gasket, J. Appl. Phys. 115 (2014) 163509.

[30] V. I. Levitas, O. M. Zarechnyy, Modeling and simulation of strain-induced phase transformations under compression and torsion in a rotational diamond anvil cell, Phys. Rev. B 82 (2010) 174124.

[31] B. Feng, V. I. Levitas, Coupled phase transformations and plastic flows under torsion at high pressure in rotational diamond anvil cell: Effect of contact sliding J. Appl. Phys. 114 (2013) 213514.

[32] B. Feng, V. I. Levitas, O. M. Zarechnyy, Strain-induced phase transformations under high pressure and large shear in a rotational diamond anvil cell: Simulation of loading, unloading, and reloading, Comp. Mater. Sci. 84 (2014) 404-416.

[33] B. Feng, V. I. Levitas, Effects of gasket on coupled plastic flow and strain-induced phase transformations under high pressure and large torsion in a rotational diamond anvil cell, J. Appl. Phys. 119 (2016) 015902.

[34] V. I. Levitas, Y. Z. Ma, J. Hashemi, M. Holtz, N. Guven, Strain-induced disorder, phase transformations, and transformation-induced plasticity in hexagonal boron nitride under compression and shear in a rotational, J. Chem. Phys. 125 (2006) 044507.

[35] V. A. Zilbershtein, N. P. Chistotina, A. A. Zharov, N. S. Grishina, E. I. Estrin, Alpha-omega transformation in titanium and zirconium during shear deformation under pressure, Fizika Metallov I Metallovedenie 39 (1975) 445-447.

[36] A. P. Zhilyaev, I. Sabirov, G. Gonzalez-Doncel, J. Molina-Aldareguia, B. Srinivasarao, M. T. Perez-Prado, Effect of $\mathrm{Nb}$ additions on the microstructure, thermal stability and mechanical behavior of high pressure $\mathrm{Zr}$ phases under ambient conditions, Mat. Sci. Eng. A. 528 (2011) 3496-3505.

[37] Y. S. Zhao, J. Z. Zhang, Enhancement of yield strength in zirconium metal through high-pressure induced structural phase transition, Appl. Phys. Lett. 91 (2007) 201907.

[38] V. A. Zilbershtein, G. I. Nosova, E. I. Estrin, Alpha--omega transformation in titanium and zirconium, Fizika Metallov I Metallovedenie 35 (1973) 584-589.

[39] M. T. Perez-Prado, A. P. Zhilyaev, First experimental observation of shear induced hcp to bcc transformation in pure Zr, Phys. Rev. Lett. 102 (2009) 175504.

[40] R. J. Hemley, H. K. Mao, G. Y. Shen, J. Badro, P. Gillet, M. Hanfland, D. Hausermann, X-ray imaging of stress and strain of diamond, iron, and tungsten at megabar pressures, Science 276 (1997) 1242-1245. 
[41] B. Feng, V. I. Levitas, R. J. Hemley, Large elastoplasticity under static megabar pressures: formulation and application to compression of samples in diamond anvil cells, Int. J. of Plast. 84 (2016) 33-57.

[42] V. I. Levitas, Large Deformation of Materials with Complex Rheological Properties at Normal and High Pressure, Nova Science Publishers, New York, 1996.

[43] V. D. Blank, E. I. Estrin, Phase Transitions in Solids under High Pressure, CRC Press, New York, 2014.

[44] V. I. Levitas, Phase transitions in elastoplastic materials: Continuum thermomechanical theory and examples of control. Part II, J. Mech. Phys. Solids 45 (1997) 1203-1222.

[45] V. I. Levitas, Phase transitions in elastoplastic materials: continuum thermomechanical theory and examples of control—part I, J. Mech. Phys. Solids 45 (1997) 923-947.

[46] V. I. Levitas, A. V. Idesman, E. Stein, Finite element simulation of martensitic phase transitions in elastoplastic materials, Int. J. Solids Struct. 35 (1998) 855-887.

[47] C. Lexcellent, Shape-memory alloys handbook, John Wiley \& Sons, Inc, Hoboken, 2013.

[48] V. I. Levitas, Phase-field theory for martensitic phase transformations at large strains, Int. J. of Plast. 49 (2013) 85-118.

[49] V. I. Levitas, B. F. Henson, L. B. Smilowitz, B. W. Asay, Solid-solid phase transformation via virtual melting significantly below the melting temperature, Phys. Rev. Lett. 92 (2004) 235702.

[50] V. I. Levitas, O. M. Zarechnyy, Numerical study of stress and plastic strain evolution under compression and shear of a sample in a rotational anvil cell, High Pressure Res. 30 (2010) 653-669.

[51] S. Banerjee, P. Mukhopadhyay, Phase Transformations: Examples from Titanium and Zirconium Alloys, Elsevier, (2007).

[52] M. P. Usikov, V. A. Zilbershtein, The orientation relationship between the $\alpha$-and $\omega$-phases of titanium and zirconium, Phys. Status Solidi A 19 (1973) 53-58.

[53] V. I. Levitas, Crystal-amorphous and crystal-crystal phase transformations via virtual melting, Phys. Rev. Lett. 95 (2005) 075701.

[54] V. I. Levitas, B. F. Henson, L. B. Smilowitz, B. W. Asay, Solid-Solid Phase Transformation via Internal Stressinduced Virtual Melting, Significantly below the Melting Temperature. Application to HMX Energetic Crystal, J. Phys. Chem. B 110 (2006) 10105-10119.

[55] K. Momeni, V. I. Levitas, A phase-field approach to nonequilibrium phase transformations in elastic solids via an intermediate phase (melt) allowing for interface stresses, Phys. Chem. Chem. Phys. 18 (2016) 12183-12203.

[56] D. Errandonea, Y. Meng, M. Somayazulu, D. Hausermann, Pressure induced $\alpha \rightarrow \omega$ transition in titanium metal: a systematic study of the effects of uniaxial stress, Physica B 355 (2005) 116-125.

[57] E. Cerreta, G. T. Gray, A. C. Lawson, T. A. Mason, C. E. Morris, The influence of oxygen content on the $\alpha$ to $\omega$ phase transformation and shock hardening of titanium, J. Appl. Phys. 100 (2006) 013530.

[58] O. Mishima, Relationship between melting and amorphization of ice, Nature 384 (1996) 546-549.

[59] O. Mishima, L. D. Calvert, E. Whalley, Nature 310 (1984) 393-395.

[60] A.P. Zhilyaev, F. Gálvez, A. Sharafutdinov, M.T. Pérez-Prado, Influence of the high pressure torsion die geometry on the allotropic phase transformations in pure Zr, Mat. Sci. Eng. A. 527 (2010) 3918-3928.

[61] K. Edalati, Z. Horita, S. Yagi, E. Matsubara, Allotropic phase transformation of pure zirconium by high-pressure torsion , Mat. Sci. Eng. A. 523 (2009) 277-281.

[62] C. Nisoli, H. Zong, S. R. Niezgoda, D. W. Brown, T. Lookman, Long-time behavior of the $\omega \rightarrow \alpha$ transition in shocked zirconium: Interplay of nucleation and plastic deformation, Acta Mater. 108 (2016) 138-142.

[63] C. H. Lu, E. N. Hahn, B. A. Remington, B. R. Maddox, E. M. Bringa, M. A. Meyers, Phase transformation in Tantalum under extreme laser deformation, Sci. Rep. 5 (2015) 15064. 